\title{
Non-abelian lattice gauge theory with a topological action
}

\author{
Daniel Nogradi, ${ }^{a, b}$ Lorinc Szikszai $^{a}$ and Zoltan Varga ${ }^{a, c}$ \\ ${ }^{a}$ Eotvos University, Department of Theoretical Physics, \\ Pazmany Peter setany 1/a, Budapest 1117, Hungary \\ ${ }^{b}$ Universidad Autonoma de Madrid, Instituto de Fisica Teorica UAM/CSIC, and \\ Departamento de Fisica Teorica, 28049 Madrid, Spain \\ ${ }^{c}$ Budapest University of Technology and Economics, Department of Theoretical Physics, \\ Budafoki ut 8, Budapest 1521, Hungary \\ E-mail: nogradi@bodri.elte.hu, szikszail@caesar.elte.hu, \\ varga.zoltan@wigner.mta.hu
}

ABSTRACT: $\mathrm{SU}(2)$ gauge theory is investigated with a lattice action which is insensitive to small perturbations of the lattice gauge fields. Bare perturbation theory can not be defined for such actions at all. We compare non-perturbative continuum results with that obtained by the usual Wilson plaquette action. The compared observables span a wide range of interesting phenomena: zero temperature large volume behavior (topological susceptibility), finite temperature phase transition (critical exponents and critical temperature) and also the small volume regime (discrete $\beta$-function or step-scaling function). In the continuum limit perfect agreement is found indicating that universality holds for these topological lattice actions as well.

KeYworDs: Lattice Quantum Field Theory, Nonperturbative Effects

ARXIV EPRINT: 1807.05295 


\section{Contents}

1 Introduction 1

2 Topological lattice action $\quad 2$

3 Topological susceptibility and $t_{0}$ scale 3

4 Deconfinement phase transition 4

5 Small volume, perturbative regime $\quad 8$

$\begin{array}{llr}6 & \text { Conclusion and outlook } & 9\end{array}$

\section{Introduction}

Universality for lattice gauge theory is generally thought to mean that any lattice action can be used in simulations provided (1) it reproduces the desired continuum action in the classical continuum limit, (2) is local, (3) has the correct symmetries, (4) the continuum extrapolation is performed. Due to asymptotic freedom bare perturbation theory then correctly predicts the approach to the continuum.

The possibility that universality in field theory holds more broadly was first addressed in the non-linear $O(3)$ model in 2 dimensions [1]. It was shown that a topological lattice action which is insensitive to small perturbations of the lattice fields, gives the correct results in the continuum. The topological nature of the lattice action means that the classical vacuum is infinitely degenerate and hence bare perturbation theory can not be set up and is inherently meaningless. The only result that may be obtained from a topological action is the fully non-perturbative one and it is apparently the same as the one with the usual lattice action of the $O(3)$ model. Hence it seems that requirement (1) above can be dropped and universality still holds. Similar results were also shown $[2,3]$ to hold for the 2d XY model as well. ${ }^{1}$

From the point of view of the path integral a useful way of thinking about the type of topological actions we investigate is the following. Field space is divided into two sets: one, where the action is zero and two, where the action is infinite. Hence configurations from the former enter with equal weights and fluctuate freely while configurations from the latter are forbidden. The only non-trivial information about the field theory is then encoded in the boundary separating the two sets.

\footnotetext{
${ }^{1} \mathrm{U}(1)$ gauge theory was studied with a topological action in $[4,5]$ but it is trivial in the physically relevant continuum limit.
} 
We investigate the same phenomenon for non-abelian gauge theories. A topological lattice action can easily be defined analogously to the $O(3)$ model and hence bare perturbation theory is again meaningless. Nevertheless we show that for $\mathrm{SU}(2)$ pure gauge theory the non-perturbative continuum results obtained with the topological action agree with that of the usual Wilson plaquette action which we know is in the correct universality class of Yang-Mills theory. The compared observables are sensitive to a wide range of interesting physics. At $T=0$ we calculate the topological susceptibility and set the scale $t_{0}$ by the gradient flow. At $T=T_{c}$ we compare the dimensionless ratio $T_{c} \sqrt{8 t_{0}}$ and also the critical exponent $1 / \nu$ from the Binder cumulant of the Polyakov loop. In small physical volumes we calculate the discrete $\beta$-function (or step-scaling function) at two values of the renormalized coupling in the gradient flow scheme. In all cases perfect agreement is found between continuum extrapolated results using the topological and Wilson plaquette gauge actions. It is worthwhile to point out that a smooth action that effectively prohibits large plaquettes and is very close to the Wilson plaquette action for small plaquettes was investigated recently in [6].

The organization of the paper is as follows. In section 2 the topological lattice action and the details of our simulations are given. Section 3 is dedicated to the topological susceptibility and scale setting, in section 4 the results related to the deconfinement phase transition are discussed and in section 5 the results for the discrete $\beta$-function are presented. Finally in section 6 we end with a conclusion and possible future aspects.

\section{Topological lattice action}

We seek an action which is gauge invariant. The simplest possibility is to use the usual plaquette $P$ as the only building block,

$$
\begin{aligned}
S & =\sum_{P} S(P) \\
S(P) & =\left\{\begin{array}{lll}
0 & \text { if } & 1-\frac{1}{2} \operatorname{Tr} P<\delta \\
\infty & \text { otherwise }
\end{array}\right.
\end{aligned}
$$

where the sum is over all plaquettes on the lattice. On a given lattice volume the only parameter is $\delta$ which will play the role of a bare coupling. Clearly, as $\delta \rightarrow 0$ only links close to unity are allowed hence $\delta \rightarrow 0$ will correspond to the continuum limit.

Note that the action (2.1) has only two values, 0 or $\infty$, hence divides the space of links into two subsets. On one, which contain the unit links, the action is zero and hence the links fluctuate freely without any weight and contribute equally to the path integral. In particular, the vacuum is infinitely degenerate. On the second set of links the action is $\infty$ meaning that links are forbidden there and contribute nothing to the path integral. The only dynamical information is carried by the boundary of these two sets defined by $\delta$. As the continuum is approached, $\delta \rightarrow 0$, links have less and less phase space to fluctuate but still always have equal weight. Gauge invariance is encoded in a gauge invariant definition of the boundary between allowed and not allowed links. 


\begin{tabular}{|c|c|c|c|c|c|c|c|}
\hline$\delta$ & $L / a$ & $t_{0} / a^{2}$ & $t_{0}^{2} \chi$ & $\beta$ & $L / a$ & $t_{0} / a^{2}$ & $t_{0}^{2} \chi$ \\
\hline 0.8022 & 20 & $1.6739(8)$ & $0.00084(3)$ & 2.2986 & 20 & $1.572(2)$ & $0.00070(5)$ \\
\hline 0.7411 & 24 & $3.512(3)$ & $0.00123(4)$ & 2.4265 & 24 & $3.364(3)$ & $0.00117(4)$ \\
\hline 0.7031 & 32 & $6.106(6)$ & $0.00135(6)$ & 2.5115 & 32 & $5.843(3)$ & $0.00139(3)$ \\
\hline 0.6792 & 40 & $8.95(1)$ & $0.00148(5)$ & 2.5775 & 40 & $8.93(1)$ & $0.00152(5)$ \\
\hline
\end{tabular}

Table 1. The scale $t_{0}$ and topological susceptibility with the topological (left 4 columns) and Wilson plaquette action (right 4 columns).

It is well-known [7] that if the plaquettes on a lattice are all restricted to be very small, $1-\frac{1}{2} \operatorname{Tr} P<0.015$, then a geometric integer definition of the topological charge $Q$ can be given. A slightly more permissive bound was later derived in [8], $1-\frac{1}{2} \operatorname{Tr} P<\frac{1}{12(2+\sqrt{2})} \simeq$ 0.0244. Hence for very small bare couplings $\delta$ a local algorithm can not change topology. In practice though the values of $\delta$ we use in this work are much larger, $\delta \sim 0.6 \ldots 0.8$, and we do encounter topology change frequently enough in all large volume runs. In section 5 the simulations are done in very small physical volumes where of course $Q=0$ but this is not an algorithmic artifact but rather the consequence of being in the femto world. Even in this case $\delta>0.3$.

In all runs with the topological action we use a simple Metropolis algorithm whereas with the Wilson plaquette action a heat bath algorithm. Both Metropolis and heat bath sweeps are accompanied by two to five overrelaxation steps [9]. An allowed configuration by the topological action may turn into a forbidden configuration by an overrelaxation step, in this case the step is rejected and the original configuration is kept.

\section{Topological susceptibility and $t_{0}$ scale}

The first observable we would like to compare in the continuum is the topological susceptibility. The gradient flow $t_{0}$ scale [10-15] is used to make it dimensionless and set the scale, hence we will compare $\chi t_{0}^{2}=\left\langle Q^{2}\right\rangle t_{0}^{2} / V$.

For both the topological action and the Wilson plaquette action the measurement of the gradient flow is done in the same way, using the plaquette discretization along the flow and the symmetric clover discretization for the observable $E(t)$,

$$
\begin{aligned}
E(t) & =-\frac{1}{2} \operatorname{Tr} F_{\mu \nu}(t) F_{\mu \nu}(t) \\
\left\langle t_{0}^{2} E\left(t_{0}\right)\right\rangle & =0.3
\end{aligned}
$$

where on the right hand side the choice 0.3 could in principle be different. Choosing a smaller value would lead to smaller finite volume effects, smaller errors but larger cut-off effects. The topological charge $Q$ is also measured along the flow at $t=t_{0}$ requiring no renormalization for the topological susceptibility. We confirmed that the continuum results are insensitive to the choice of $t$ as long as it is kept fixed in physical units. For example, using $t=0.75 t_{0} \ldots 1.25 t_{0}$ for the susceptibility leads to identical results. 


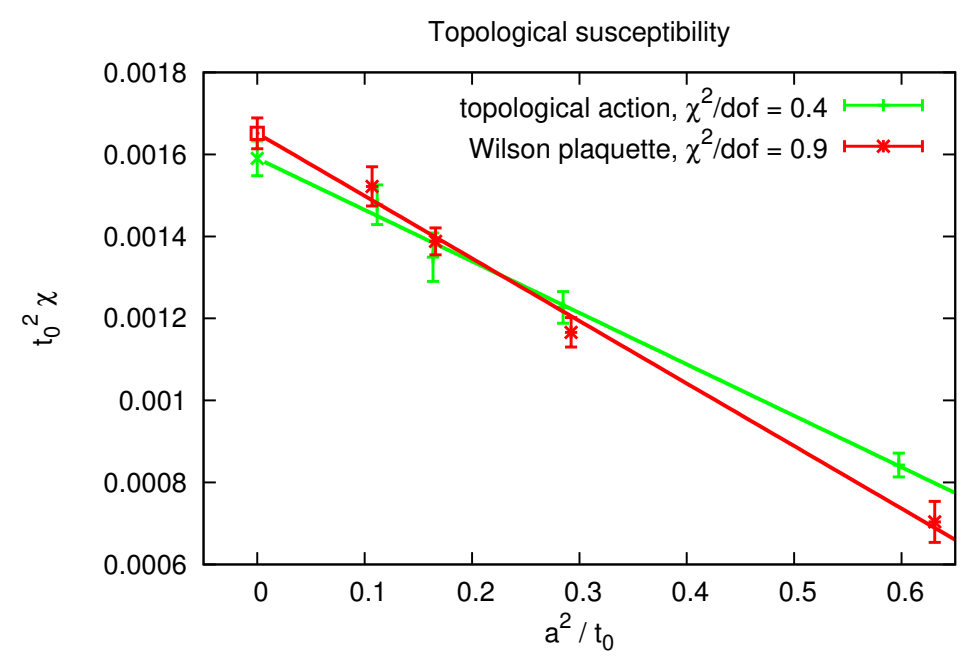

Figure 1. Continuum extrapolations of the topological susceptibility.

Finite volume effects are ensured to be negligible within our statistical errors by always using symmetric $L^{4}$ lattices such that $\sqrt{8 t_{0}} / L<0.25$. Another indicator that finite volume effects are small is that we always have $T_{c} L \geq 4$ (see next section).

The measurement of $t_{0} / a^{2}$ in our simulations is very precise, its relative error is at least an order of magnitude smaller than the relative error on the susceptibility. Hence the final errors are completely dominated by the latter. The data is shown in table 1 , the generated number of configurations at each point is $O\left(10^{5}\right)$ with $O(100)$ configurations separating the measurements.

The continuum extrapolation of $t_{0}^{2} \chi$ in $a^{2} / t_{0}$ is straightforward and shown in figure 1 . The continuum results for the two discretizations agree, 0.00159(4) and 0.00165(4) for the topological and Wilson plaquette actions, respectively.

\section{Deconfinement phase transition}

Next we compare quantities which are sensitive to the deconfinement phase transition which is second order for $\mathrm{SU}(2)$. These can again be compared to the results obtained with the Wilson plaquette gauge action or with the corresponding quantities in the 3dimensional Ising model. First, using the Binder cumulant of the Polyakov loop we will determine the critical exponent $1 / \nu$ with the topological action and find that it agrees with $1 / \nu=1.5878$ (4) from the 3-dimensional Ising model [16]. Then the critical couplings are determined on $N_{t}=4,6,8,10$ lattices and the dimensionless ratio $T_{c} \sqrt{8 t_{0}}$ is obtained in the continuum. Again perfect agreement is found between the two actions.

We will use standard scaling theory for much of this section; see [17] for more details. The bare parameters, $\delta$ or $\beta$, are collectively denoted by $\alpha$. Since we investigate the deconfinement phase transition at fixed temporal extent, the dependence on $N_{t}$ is often suppressed while the spatial volume is denoted by $N_{s}$. The Binder cumulant of the Polyakov 

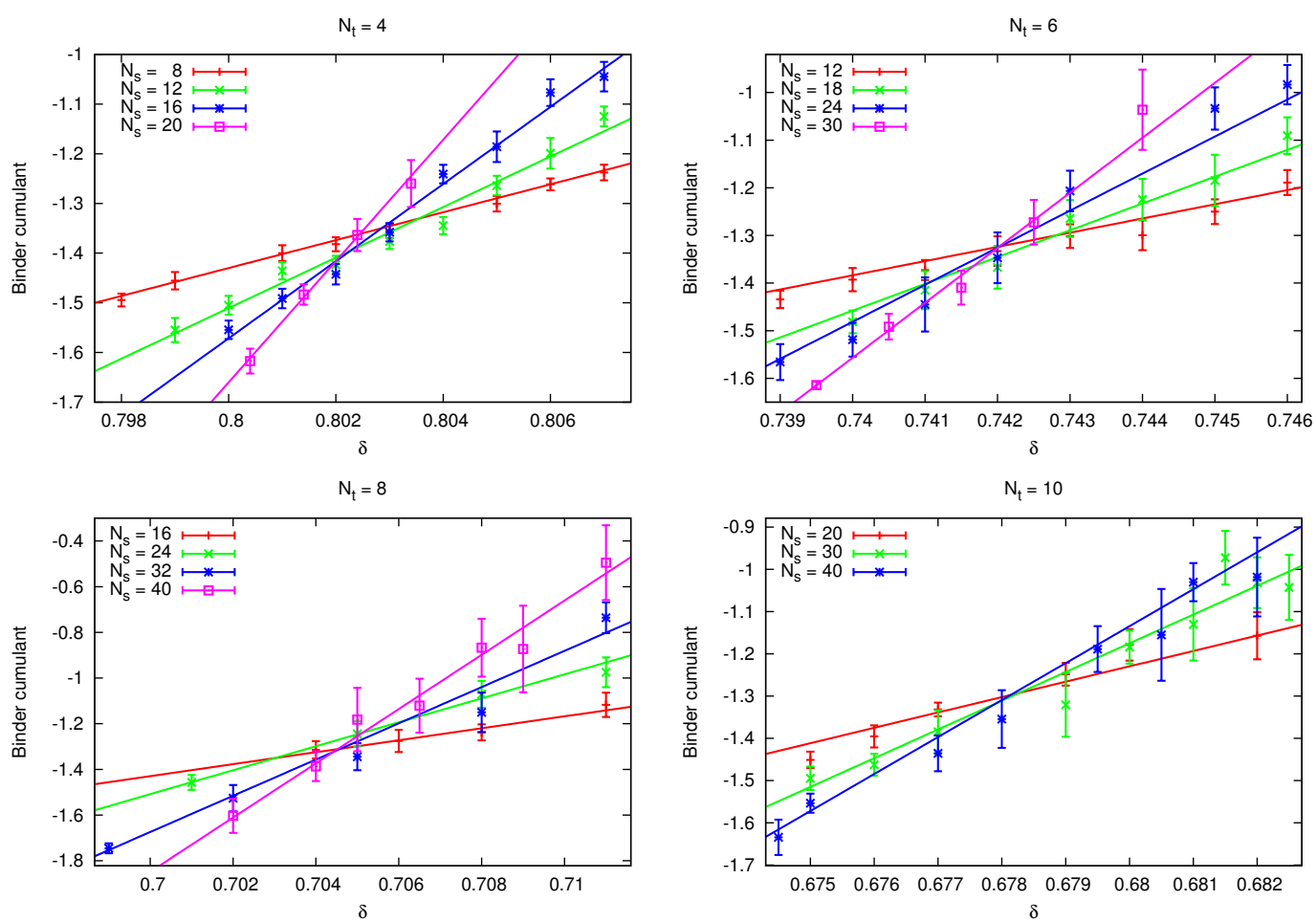

Figure 2. The measured Binder cumulants of the Polyakov loop and linear interpolations close to the critical points with the topological action.

loop $L$ is defined by

$$
g\left(\alpha, N_{s}\right)=\frac{\left\langle L^{4}\right\rangle}{\left\langle L^{2}\right\rangle^{2}}-3
$$

The reduced temperature will be denoted by $x=\left(\alpha-\alpha_{c}\right) / \alpha_{c}$. Scaling theory says that close to the critical point a scaling function $f$ can be defined such that $g\left(\alpha, N_{s}\right)=f\left(x N_{s}^{1 / \nu}\right)$ with some critical exponent $\nu$. Expanding around $x=0$ we obtain

$$
g\left(\alpha, N_{s}\right)=f(0)+f^{\prime}(0) x N_{s}^{1 / \nu}+\ldots
$$

which means that by considering two spatial volumes, $N_{s}$ and $b N_{s}$, for some $b>1$, we may estimate the exponent as

$$
\frac{1}{\nu}=\frac{\log \left(\frac{S\left(b N_{s}\right)}{S\left(N_{s}\right)}\right)}{\log (b)},
$$

where $S\left(N_{s}\right)$ is the slope of $g\left(\alpha, N_{s}\right)$ with respect to $\alpha$ close to the critical point.

Our data for the Binder cumulant for $N_{t}=4,6,8,10$ are shown in figure 2 together with linear interpolations (the largest $\chi^{2} /$ dof is 1.55). The generated number of configurations is $O\left(10^{5}\right)-O\left(10^{6}\right)$, depending on the parameters. The slopes of these interpolations directly give an estimate of the critical exponent $1 / \nu$ via equation (4.3) without the need to know the precise location of $\alpha_{c}$. The results are given in table 2. Clearly, the expected exponent 


\begin{tabular}{|c|c|c|c|}
\hline$N_{t}$ & $N_{s}$ & $b$ & $1 / \nu$ \\
\hline 4 & 12 & $5 / 3$ & $1.71(25)$ \\
\hline 6 & 18 & $5 / 3$ & $1.40(20)$ \\
\hline 8 & 24 & $5 / 3$ & $1.60(29)$ \\
\hline 10 & 20 & $3 / 2$ & $1.54(29)$ \\
\hline
\end{tabular}

Table 2. The critical exponent $1 / \nu$ with the topological action from $N_{t}=4,6,8,10$ lattices.

\begin{tabular}{|c|c|c|c|c|}
\hline$N_{t}$ & $\delta_{c}$ & $t_{0} / a^{2}$ & $\beta_{c}$ & $t_{0} / a^{2}$ \\
\hline 4 & $0.8015(5)$ & $1.68(2)$ & $2.2986(6)$ & $1.572(5)$ \\
\hline 6 & $0.7413(8)$ & $3.53(3)$ & $2.4265(30)$ & $3.36(7)$ \\
\hline 8 & $0.705(2)$ & $6.05(9)$ & $2.5115(40)$ & $5.8(2)$ \\
\hline 10 & $0.678(3)$ & $9.2(3)$ & $2.5775(24)$ & $8.9(1)$ \\
\hline
\end{tabular}

Table 3. Infinite volume extrapolated critical couplings and $t_{0}$ scale for both the topological and Wilson actions. The critical $\beta_{c}$ for $N_{t}=4,6,8$ are from [17]. The errors on $t_{0} / a^{2}$ contain the error of the critical couplings, that is why these errors are larger than those in table 1 . The 4 -volume for the scale measurements are the same as in table 1.

of the 3D Ising model, $1 / \nu=1.5878(4)$, is consistent with our data at each $N_{t}$ even at finite $N_{s}$.

Next, we turn to the determination of the critical couplings. Let us denote the intersection of the Binder cumulants corresponding to $N_{s}$ and $b N_{s}$ by $\alpha\left(N_{s}, b\right)$. The dependence on $N_{s}$ and $b$ is again fixed by scaling theory and for large enough volumes we have, with some constant $A$,

$$
\begin{aligned}
& \alpha\left(N_{s}, b\right)=\alpha_{c}+A \varepsilon\left(N_{s}, b\right) \\
& \varepsilon\left(N_{s}, b\right)=\frac{1}{N_{s}^{-y_{1}+1 / \nu}} \frac{1-b^{y_{1}}}{b^{1 / \nu}-1},
\end{aligned}
$$

where $y_{1}=-1$ for the $3 \mathrm{D}$ Ising model. Having established that our result for $1 / \nu$ is compatible with the 3D Ising model, we will simply use $y_{1}=-1$ and $1 / \nu=1.5878$ in the above infinite volume extrapolations.

The infinite volume extrapolations, using (4.4) is shown in figure 3. Estimating the statistical error on $\alpha_{c}$ is non-trivial since the pair-wise intersections for various $N_{s}$ are correlated; we use a jackknife procedure starting from the independently measured Binder cumulants.

The critical couplings with the Wilson plaquette action have been determined in [17] for $N_{t}=4,6,8$. Our results for the Binder cumulants and infinite volume extrapolation for $N_{t}=10$ is shown in figure 4 .

The final results for the critical couplings are listed in table 3 which we subsequently use to determine $T_{c}$ in physical units, $T_{c} \sqrt{8 t_{0}}$.

In order to determine the dimensionless combination $T_{c} \sqrt{8 t_{0}}$ in the continuum only $t_{0} / a^{2}$ needs to be measured at the critical couplings. Even though the statistical error of $t_{0} / a^{2}$ is very small in a given simulation, the uncertainty of the critical coupling itself needs 

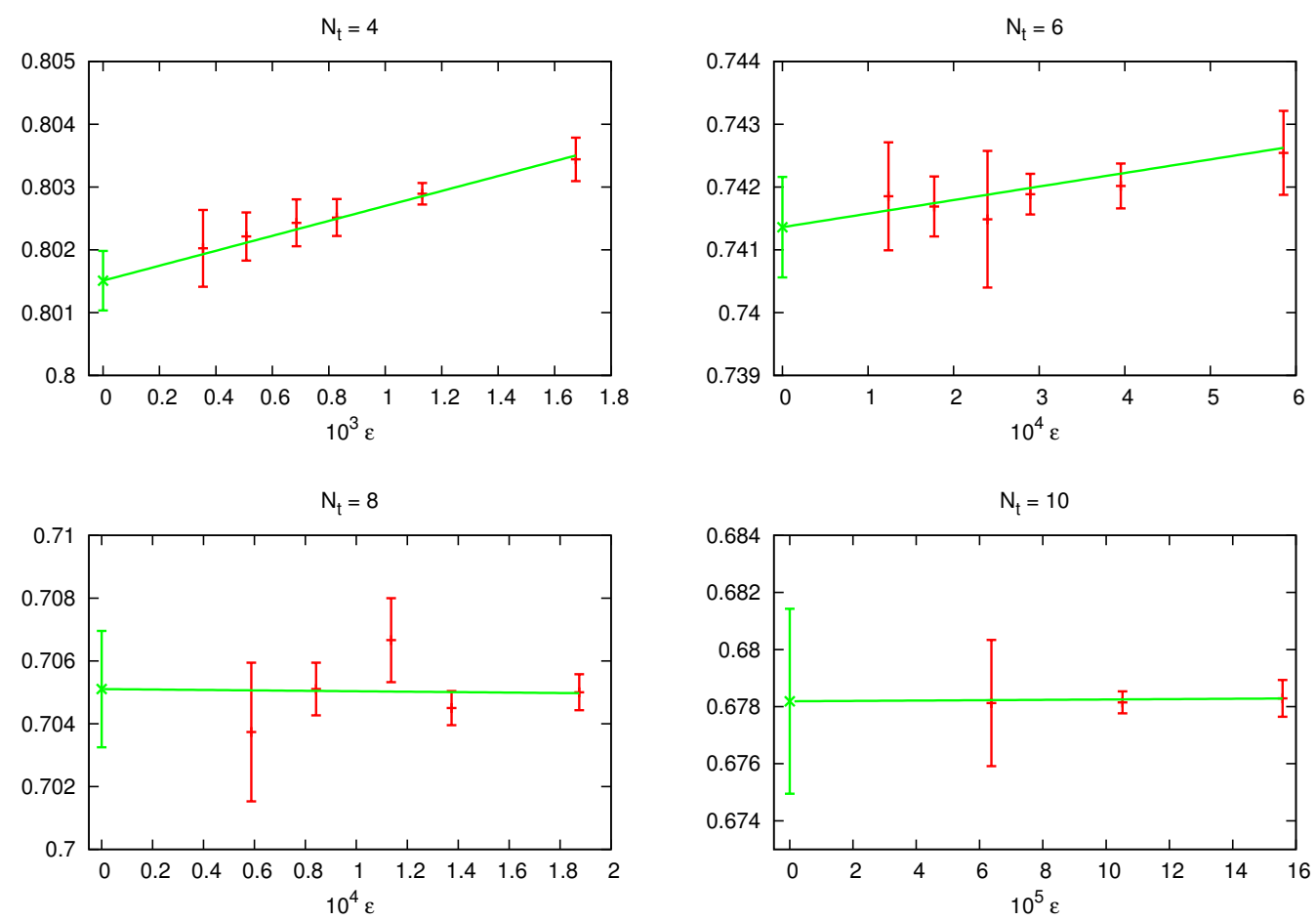

Figure 3. Infinite volume extrapolations of the critical couplings with the topological action; see equation (4.4).
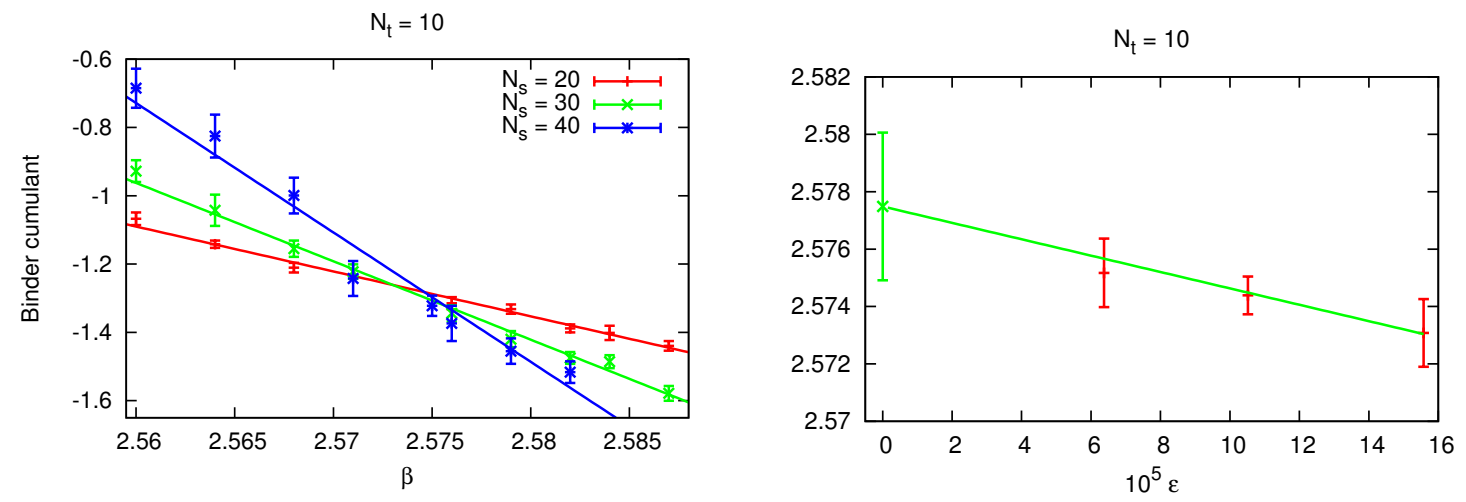

Figure 4. Binder cumulants and infinite volume extrapolation of the critical coupling with the Wilson plaquette action at $N_{t}=10$.

to be taken into account. By a simple interpolation of $t_{0} / a^{2}$ in $\delta$ and $\beta$ we estimate this uncertainty originating from the uncertainty on the critical couplings. It turns out that this is the dominant source of final uncertainty, the statistical error is negligible. In table 3 we list the results and the reason for the unusually large error on $t_{0} / a^{2}$, relative to table 1 , is the aforementioned effect. Another way of saying it is that if only the central values of $\beta_{c}$ and $\delta_{c}$ are taken, then there is an uncertainty on $N_{t}$ leading to an additional uncertainty on $\sqrt{8 t_{0} / a^{2}} / N_{t}$ beside $t_{0} / a^{2}$. 


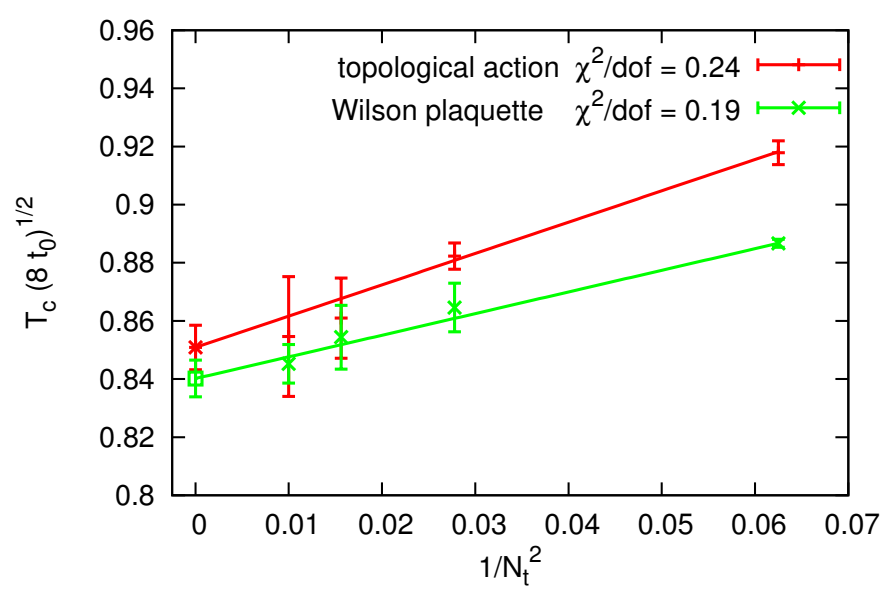

Figure 5. Continuum extrapolation of $T_{c} \sqrt{8 t_{0}}$.

The continuum extrapolation is shown in figure 5, the results are $0.851(8)$ and $0.840(6)$ with the topological and Wilson plaquette action, respectively. Again, complete agreement is found.

\section{Small volume, perturbative regime}

In this section we compare quantities in the perturbative regime. The simplest example is given by the discrete $\beta$-function or step-scaling function [18] in small physical volume or femto world [19-21] We will use the finite volume gradient flow scheme with periodic boundary conditions $[22,23]$. In this scheme the renormalized coupling is defined by

$$
g_{R}^{2}(L)=\frac{128 \pi^{2}}{9(1+\delta(c))}\left\langle t^{2} E(t)\right\rangle
$$

where $c=\sqrt{8 t} / L$ is a constant so $g_{R}^{2}$ only depends on one scale, $L$, the linear size of the periodic box. The factor

$$
\delta(c)=-\frac{c^{4} \pi^{2}}{3}+\vartheta^{4}\left(e^{-1 / c^{2}}\right)-1
$$

is such that at tree level the above scheme agrees with $\overline{\mathrm{MS}}, \vartheta$ is the 3rd Jacobi elliptic function $[22,23]$. We set $c=3 / 10$. The boundary condition is periodic in all directions and it is well-known that there are degenerate perturbative vacua within this setup. However for small renormalized couplings the system fluctuates around one of the vacua and tunnelling events are suppressed. In our simulations we work in this regime and indeed do not detect tunnelling at all as expected.

The expansion of $g_{R}^{2}(L)$ in terms of $g_{\overline{\mathrm{MS}}}$ is unusual in a periodic 4 -torus in the sense that both even and odd powers can appear, as well as non-analytic logarithms [21]. Particularly for $\mathrm{SU}(2)$ purely logarithmically suppressed terms appear right after tree level; see [22] for an extended discussion. Hence only the first coefficient of the $\beta$-function is the same in the gradient flow scheme and $\overline{\mathrm{MS}}$. It is important to note that the scheme as such is completely 
well-defined non-perturbatively, merely the perturbative expansion behaves in a somewhat unusual way. The $\beta$-function at fixed $g_{R}^{2}$ is a perfectly well-defined and universal quantity. The subscript $R$ will be dropped in what follows.

On the lattice the discrete $\beta$-function, $\left(g^{2}(s L)-g^{2}(L)\right) / \log \left(s^{2}\right)$, or step-scaling function is the most easily accessible quantity with a well-defined continuum limit for fixed $g^{2}(L)$. We set $s=3 / 2$. It has a perturbative expansion

$$
\frac{g^{2}(s L)-g^{2}(L)}{\log \left(s^{2}\right)}=b_{0} \frac{g^{4}(L)}{16 \pi^{2}}+\ldots
$$

where, as mentioned above, the terms in ... contain both even and odd powers of $g(L)$ as well as logarithms. The 1-loop term $b_{0}=22 / 3$ is nevertheless universal.

We computed the discrete $\beta$-function at two values of the renormalized coupling, $g^{2}(L)=1.5$ and $g^{2}(L)=2.5$. As we will see the first is small enough such that the continuum result is compatible with the 1-loop approximation (5.3). The continuum limit is approached by 3 sets of lattice volumes, $16^{4} \rightarrow 24^{4}, 18^{4} \rightarrow 27^{4}$ and $24^{4} \rightarrow 36^{4}$ corresponding to the scale change $s=3 / 2$. The desired values of $g^{2}(L)$ were tuned on the smaller lattices to high accuracy by tuning the bare couplings of the two actions, $\beta$ and $\delta$, respectively. Then at the same values of the bare couplings the renormalized couplings were measured on the larger lattices. Finally the results were extrapolated to the continuum linearly in $a^{2} / L^{2}$.

The tuned couplings and the measured values on the larger lattices are shown in table 4. The continuum extrapolations are shown in figure 6 , all $\chi^{2} / d o f$ are less than unity. As emphasized above the 2-loop result is only shown for orientation, it is not universal in our scheme. There is perfect agreement for the continuum results between the two actions. The smaller coupling, $g^{2}(L)=1.5$ is expected to be small enough such that renormalized perturbation theory at 1-loop is trustworthy. This seems to be the case despite the only logarithmically suppressed terms and the results with the Wilson plaquette and topological actions are compatible with the 1-loop approximation (5.3) within $1 \sigma$ and $1.3 \sigma$, respectively. Even though bare perturbation theory is not possible to set up, renormalized perturbation theory behaves as expected.

\section{Conclusion and outlook}

In this paper we investigated non-abelian lattice gauge theory with an action that is insensitive to small perturbations of the lattice fields. In particular, the classical vacuum is infinitely degenerate. By comparing continuum extrapolated results to those obtained with the Wilson plaquette action (which is known to be in the correct universality class) we conclude that even though the topological action has no classical continuum limit, the quantum continuum limit correctly reproduces the theory. There are of course only a finite number of comparisons that one can do in simulations but we have chosen quantities that span a wide range of interesting phenomena. The topological susceptibility, the critical temperature, critical exponents, and the $\beta$-function in small physical volumes were compared and perfect agreement was found. This suggests that universality on the lattice 


\begin{tabular}{|c|c|c|c|c|c|c|}
\hline$L / a$ & $\delta$ & $g^{2}(L)$ & $g^{2}(s L)$ & $\delta$ & $g^{2}(L)$ & $g^{2}(s L)$ \\
\hline 16 & 0.34174 & $1.498(2)$ & $1.604(2)$ & 0.44743 & $2.502(2)$ & $2.809(5)$ \\
\hline 18 & 0.33798 & $1.500(2)$ & $1.602(2)$ & 0.44049 & $2.499(2)$ & $2.799(5)$ \\
\hline 24 & 0.32907 & $1.502(2)$ & $1.601(3)$ & 0.42603 & $2.502(3)$ & $2.781(7)$ \\
\hline$L / a$ & $\beta$ & $g^{2}(L)$ & $g^{2}(s L)$ & $\beta$ & $g^{2}(L)$ & $g^{2}(s L)$ \\
\hline 16 & 4.68515 & $1.500(1)$ & $1.609(1)$ & 3.65284 & $2.500(2)$ & $2.806(2)$ \\
\hline 18 & 4.73710 & $1.500(2)$ & $1.604(1)$ & 3.70290 & $2.500(2)$ & $2.794(3)$ \\
\hline 24 & 4.86235 & $1.500(2)$ & $1.598(2)$ & 3.82180 & $2.502(2)$ & $2.777(4)$ \\
\hline
\end{tabular}

Table 4. Renormalized couplings, top 3 rows: topological action, bottom 3 rows: Wilson plaquette action. Left 3 columns: tuned to $g^{2}(L)=1.5$, right 3 columns: tuned to $g^{2}(L)=2.5$.
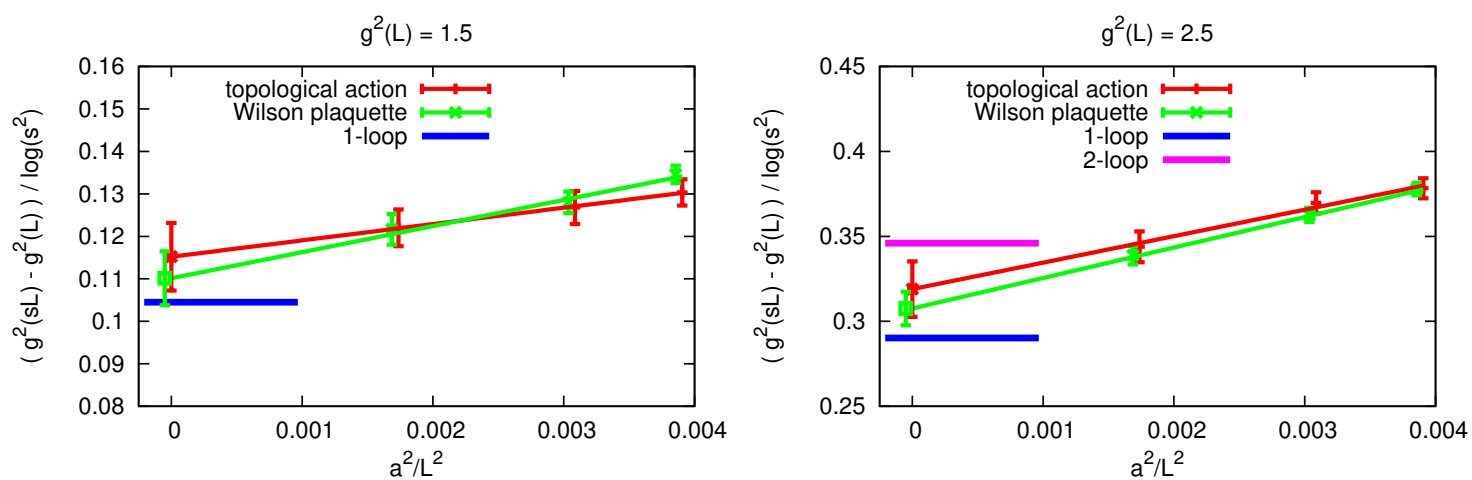

Figure 6. Continuum extrapolations of the discrete $\beta$-function at $g^{2}(L)=1.5$ (left) and $g^{2}(L)=2.5$ (right). The Wilson plaquette data is displaced slightly for better visibility.

is very robust; one is free to modify the lattice action not only with higher dimensional irrelevant operators but also the relevant operators do not have to be reached in a smooth way dictated by the classical continuum Lagrangian.

It may be worthwhile to remember that a positive transfer matrix can not be associated with a topological action at finite lattice spacing [24]. However, as duly pointed out in [24], this is not necessarily a problem if the effect of non-positivity disappears in the continuum limit. In other words if the positivity violation is merely a cut-off effect. Our results indicate that this is indeed the case since in the continuum the correct Euclidean Yang-Mills theory is obtained.

A less obvious question concerns the form of scaling violations, $O\left(a^{2}\right)$ specifically, confirmed numerically in our work. Since bare perturbation theory is not applicable it is not immediately clear that the same scaling is expected as with the Wilson plaquette action. However, following the same line of reasoning as for the 2-dimensional $O(3)$ model in [1], Symanzik's effective theory, formulated in the continuum, still applies and leads to the same $O\left(a^{2}\right)$ scaling violations as with the Wilson plaquette action.

An intriguing application of topological actions might be with dynamical fermions. The Nielsen-Ninomiya no-go theorem [25] heavily relies on the classical action, namely that in 
momentum space the continuum Dirac operator is reproduced in the classical continuum limit. With a topological fermionic action the classical continuum limit is meaningless. Hence perhaps the no-go theorem may be circumvented by such actions although it is of course not at all clear how they would be first defined and then implemented.

\section{Acknowledgments}

DN would like to thank the Universidad Autonoma de Madrid for hospitality where parts of this work was done.

Open Access. This article is distributed under the terms of the Creative Commons Attribution License (CC-BY 4.0), which permits any use, distribution and reproduction in any medium, provided the original author(s) and source are credited.

\section{References}

[1] W. Bietenholz, U. Gerber, M. Pepe and U.J. Wiese, Topological lattice actions, JHEP 12 (2010) 020 [arXiv: 1009.2146] [INSPIRE].

[2] W. Bietenholz et al., Topological lattice actions for the 2d XY model, JHEP 03 (2013) 141 [arXiv: 1212.0579] [INSPIRE].

[3] W. Bietenholz, U. Gerber and F.G. Rejón-Barrera, Berezinskii-Kosterlitz-Thouless transition with a constraint lattice action, J. Stat. Mech. 1312 (2013) P12009 [arXiv:1307.0485] [INSPIRE].

[4] O. Akerlund and P. de Forcrand, U(1) lattice gauge theory with a topological action, JHEP 06 (2015) 183 [arXiv: 1505.02666] [INSPIRE].

[5] P. de Forcrand and O. Akerlund, Aspects of topological actions on the lattice, PoS (LATTICE 2015) 169 [arXiv: 1601.03905] [INSPIRE].

[6] D. Banerjee et al., An improved single-plaquette gauge action, JHEP 03 (2016) 116 [arXiv: 1512.04984] [INSPIRE].

[7] M. Lüscher, Topology of lattice gauge fields, Commun. Math. Phys. 85 (1982) 39 [INSPIRE].

[8] H. Neuberger, Bounds on the Wilson Dirac operator, Phys. Rev. D 61 (2000) 085015 [hep-lat/9911004] [INSPIRE].

[9] M. Creutz, Overrelaxation and Monte Carlo Simulation, Phys. Rev. D 36 (1987) 515 [INSPIRE].

[10] R. Narayanan and H. Neuberger, Infinite $N$ phase transitions in continuum Wilson loop operators, JHEP 03 (2006) 064 [hep-th/0601210] [INSPIRE].

[11] M. Lüscher, Trivializing maps, the Wilson flow and the HMC algorithm, Commun. Math. Phys. 293 (2010) 899 [arXiv: 0907.5491] [INSPIRE].

[12] M. Lüscher, Properties and uses of the Wilson flow in lattice QCD, JHEP 08 (2010) 071 [Erratum ibid. 03 (2014) 092] [arXiv: 1006 . 4518] [INSPIRE].

[13] M. Lüscher, Topology, the Wilson flow and the HMC algorithm, PoS(LATTICE 2010) 015 [arXiv: 1009.5877] [INSPIRE]. 
[14] R. Lohmayer and H. Neuberger, Continuous smearing of Wilson Loops, PoS (LATTICE 2011) 249 [arXiv:1110.3522] [INSPIRE].

[15] M. Lüscher and P. Weisz, Perturbative analysis of the gradient flow in non-abelian gauge theories, JHEP 02 (2011) 051 [arXiv:1101.0963] [INSPIRE].

[16] M. Hasenbusch, K. Pinn and S. Vinti, Critical exponents of the three-dimensional Ising universality class from finite-size scaling with standard and improved actions, Phys. Rev. B 59 (1999) 11471 [hep-lat/9806012] [INSPIRE].

[17] J. Fingberg, U.M. Heller and F. Karsch, Scaling and asymptotic scaling in the $\mathrm{SU}(2)$ gauge theory, Nucl. Phys. B 392 (1993) 493 [hep-lat/9208012] [InSPIRE].

[18] M. Lüscher, P. Weisz and U. Wolff, A numerical method to compute the running coupling in asymptotically free theories, Nucl. Phys. B 359 (1991) 221 [INSPIRE].

[19] M. Lüscher, Some analytic results concerning the mass spectrum of Yang-Mills gauge theories on a torus, Nucl. Phys. B 219 (1983) 233 [INSPIRE].

[20] J. Koller and P. van Baal, A rigorous nonperturbative result for the glueball mass and electric flux energy in a finite volume, Nucl. Phys. B 273 (1986) 387 [INSPIRE].

[21] A. Coste et al., Zero momentum contribution to Wilson loops in periodic boxes, Nucl. Phys. B 262 (1985) 67 [INSPIRE].

[22] Z. Fodor et al., The gradient flow running coupling scheme, PoS(LATTICE 2012) 050 [arXiv: 1211.3247] [INSPIRE].

[23] Z. Fodor et al., The Yang-Mills gradient flow in finite volume, JHEP 11 (2012) 007 [arXiv:1208.1051] [INSPIRE].

[24] M. Creutz, Positivity and topology in lattice gauge theory, Phys. Rev. D 70 (2004) 091501 [hep-lat/0409017] [INSPIRE].

[25] H.B. Nielsen and M. Ninomiya, Absence of Neutrinos on a Lattice. 1. Proof by Homotopy Theory, Nucl. Phys. B 185 (1981) 20 [Erratum ibid. B 195 (1982) 541] [INSPIRE]. 